\title{
Automated extraction of UV counterterms for arbitrary local operators
}

\section{Ben Ruijl*}

Institute for Theoretical Physics, ETH Zurich, Wolfgang-Pauli-Str. 27, 8093 Zürich, CH

E-mail: bruijleethz.ch

We sketch how the $R^{*}$-operation can be used to compute the pole terms of Feynman diagrams. As an example, we briefly discuss the computatation of the Higgs decay to gluons. Next, we illustrate how $\overline{\mathrm{MS}}$ counterterms can be obtained with the $R^{*}$-operation. Finally, we describe how the UV counterterm of any $L$-loop Feynman diagram with arbitrary local operators can be computed from $L-1$-loop massless propagator diagrams.

Loops and Legs in Quantum Field Theory (LL2018)

29 April 2018 - 04 May 2018

St. Goar, Germany

${ }^{*}$ Speaker. 


\section{Introduction}

Many physically interesting quantities can be computed from the UV counterterms of Feynman diagrams, for example, anomalous dimensions (such as the beta function). The UV counterterms are part of the pole structure of a diagram, which are often much easier to compute than the finite part.

In this work we sketch the $R^{*}$-operation [1-3], which is able to compute the pole parts of $L$ loop diagrams by a computation of at most $(L-1)$-loop diagrams. Using a high-performance implementation of the $R^{*}$-operation combined with the FORCER program [4-6], we are able to compute the poles of five-loop massless propagator diagrams. Our programs rely heavily on FORM [7-10].

Using this setup, the five-loop beta function for Yang-Mills theory with fermions [11] can be computed in six days on one 32-core machine. We have also computed the $R$-ratio in $e^{+} e^{-} \rightarrow$ hadrons, and the Higgs decays to bottom quarks and gluons at five loops [12].

In this work we describe how to compute UV counterterms in $\overline{\mathrm{MS}}$ using the $R^{*}$-operation. We provide an abstract notation for extended Feynman diagrams, which can also describe derivatives and local operators. Using these two components, we can compute the UV counterterm of any $L$ loop Feynman diagram with arbitrary local operators using only $L-1$-loop massless propagators diagrams. These can automatically be computed using FORCER.

The outline of this paper is as follows. In section 2 we very briefly describe the $R^{*}$-operation. In section 3 we briefly discuss some recent physics results obtained by using the $R^{*}$-program. In section 4 we discuss how to compute UV counterterms in $\overline{\mathrm{MS}}$. In section 5 we generalize our procedure to include Feynman diagrams with arbitrary local operators. Finally, we summarize and present a brief outlook in 6 .

\section{The $R^{*}$-operation}

The $R^{*}$-operation can be used to compute the poles of Feynman diagrams [1,2]. Recently, it has been extended to Feynman diagrams with arbitrary numerator structure [3]. In this section we briefly sketch how the $R^{*}$-operation works, focusing on UV-counterterms.

The basic object of the $R^{*}$-operation is the UV counterterm operation $\Delta$ acting on a graph $G$, which is defined as the poles of $G$ in the limit of all loop momenta going to infinity with all contributions from subdivergences subtracted. Additionally, we define the pole operator $K$ for a Laurent series in $\varepsilon$

$$
K \sum_{i=-\infty}^{\infty} c_{i} \varepsilon^{i}=\sum_{i=-\infty}^{-1} c_{i} \varepsilon^{i}
$$

Then the $R^{*}$ operation for some simple examples yields

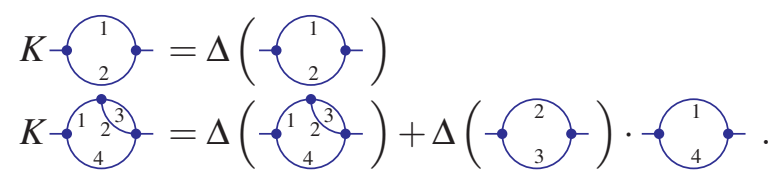


In general, all sets of non-overlapping divergent subdiagrams have to be considered:

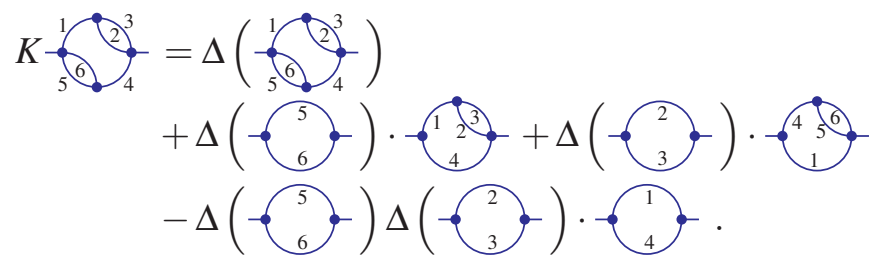

Here the contribution from two counterterms gets a minus sign, to prevent double counting.

For logarithmically divergent diagrams, $\Delta$ does not depend on external momenta or masses. Consequently, we can infrared rearrange (IRR) [13]:

$$
\Delta(\vartheta)=\Delta(\longmapsto)=\Delta(\longmapsto) .
$$

Using IRR and the definition of $\Delta$, we can express the UV-counterterm of $G$ in terms of simpler diagrams:

$$
\Delta(G) \stackrel{\text { IRR }}{=} \underbrace{\Delta\left(G^{\prime}\right)}_{\text {Simpler than } \mathrm{G}}=K\left(G^{\prime}\right)-\underbrace{\text { subdivergences }\left(G^{\prime}\right)}_{\text {Lower-loop diagrams }}
$$

Using this setup, we can rewrite all $L$-loop diagrams to $(L-1)$-loop scalar massless propagator integrals. For five-loop applications, all those integrals can be computed using FORCER [5, 6].

\section{Five-loop results}

In this section we briefly discuss the five-loop results for the Higgs-boson decay to gluons. We have used the $R^{*}$-operation to compute the pole parts, and used FORCER for the computation of the integrals. With the same setup, we have also computed the Higgs decay to bottom quarks and the electromagnetic $R$-ratio at five loops [12]. Since these were re-computations, see ref. [14] and ref. [15] and references therein, respectively, we will not address these results here.

In the heavy-top limit, there is an effective coupling of the Higgs boson to gluons. If the light-quark masses are neglected the Higgs decay to hadrons can be expressed, using the optical theorem, as

$$
\Gamma_{H \rightarrow g g}=\frac{\sqrt{2} G_{\mathrm{F}}}{M_{H}}\left|C_{1}\right|^{2} \operatorname{Im} \Pi^{G G}\left(-M_{H}^{2}-i \delta\right) .
$$

The Wilson coefficient $C_{1}$, which includes the top-mass (scheme) dependence, is known to $\mathrm{N}^{4} \mathrm{LO}$, see ref. [16] and reference therein. Due to the analytical continuation from the spacelike case, only the pole part of Higgs-boson self-energy $\Pi^{G G}$ induced by the effective $\mathrm{Hgg}$ coupling is required:

$$
\operatorname{Im} \Pi^{G G}\left(-q^{2}-i \delta\right)=\sin (L \pi \varepsilon) \Pi^{G G}\left(q^{2}\right)=\Pi^{G G}\left(q^{2}\right) L \pi \varepsilon+\ldots,
$$

where $D=4-2 \varepsilon$ and $L$ is the number of loops. Consequently, $\operatorname{Im} \Pi^{G G}\left(-q^{2}-i \delta\right)$ and hence the $\mathrm{N}^{4} \mathrm{LO}$ decay rate can be computed using the setup described above. The computation is much more costly than that of the beta function and took almost two months on the machines available to us.

For an on-shell top mass of $173 \mathrm{GeV}$, the perturbative expansion of the decay width reads

$$
\Gamma_{H \rightarrow g g}^{\overline{\mathrm{MS}}}=\Gamma_{0}\left(1+5.703052 \alpha_{\mathrm{s}}+15.51204 \alpha_{\mathrm{s}}^{2}+12.6660 \alpha_{\mathrm{s}}^{3}-69.3287 \alpha_{\mathrm{s}}^{4}+\ldots\right)
$$



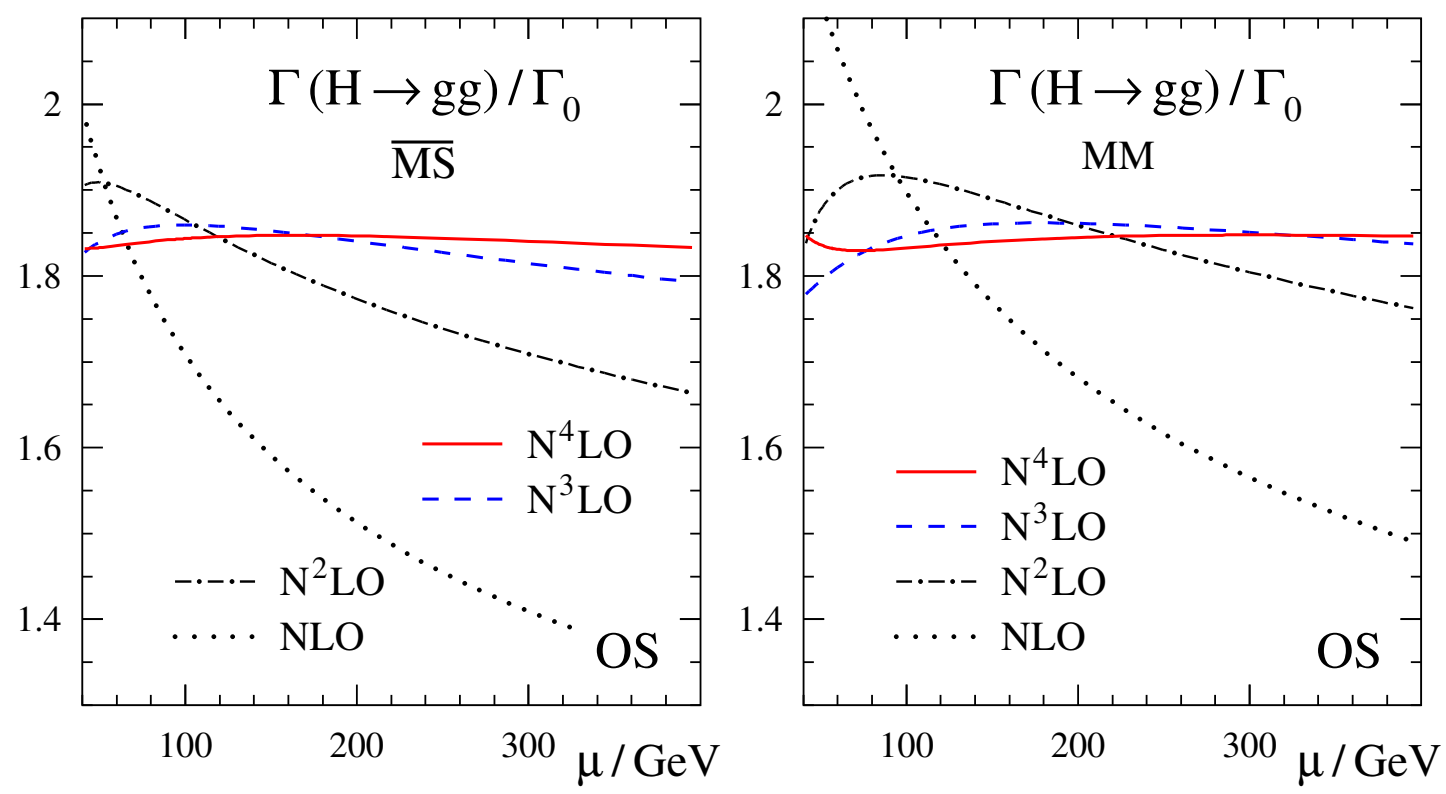

Figure 1: The renormalization scale dependence of $\Gamma_{H \rightarrow g g}$, normalized as described in the text, in the $\overline{\mathrm{MS}}$ and MiniMOM schemes, for $\alpha_{\mathrm{s}}\left(M_{Z}\right)=0.118$ in $\overline{\mathrm{MS}}, M_{H}=125 \mathrm{GeV}$ and an on-shell top-mass in $173 \mathrm{GeV}$.

at $n_{f}=5$ for the renormalization scale $\mu=M_{H}$ with $\left(G_{F}\right.$ is the Fermi constant $)$

$$
\Gamma_{0}=G_{F} M_{H}^{3} /\left(36 \pi^{3} \sqrt{2}\right) \cdot\left(\alpha_{\mathrm{s}}\left(M_{H}^{2}\right)\right)^{2} .
$$

Taking into account also the renormalization scale dependence as shown in fig. 1, we arrive at

$$
\Gamma_{\mathrm{N}^{4} \mathrm{LO}}(H \rightarrow g g)=\Gamma_{0}\left(1.844 \pm 0.011_{\text {series }} \pm 0.045_{\alpha_{\mathrm{s}}\left(M_{Z}\right), 1 \%}\right)
$$

with $\alpha_{\mathrm{s}}\left(M_{H}\right)=0.11264$, the $\overline{\mathrm{MS}}$ value corresponding to $\alpha_{\mathrm{s}}\left(M_{Z}\right)=0.118$, in eq. (3.4). For $1 / m_{\text {top }}$ corrections and light-quark mass effects, see ref. [17] and references therein.

\section{The $R^{*}$-operation and $\overline{\mathrm{MS}}$ counterterms}

In the results described above the $R^{*}$-operation has been used to compute the singular part of five-loop Feynman graphs. The pole parts are a scheme independent quantity. While computing the pole parts provides the advantage of not having to worry about the UV counterterms being in $\overline{\mathrm{MS}}$, the disadvantage is that it requires the computation of subgraphs of the non-infrared rearranged graph:

$$
K(G) \stackrel{\text { IRR }}{=} \Delta\left(G^{\prime}\right)-\text { subdivergences }\left(G^{\prime}\right)+\text { subdivergences }(G)
$$

If $G$ contains complicated local operators, it may be difficult to compute its subdivergences.

Thus the question arises if we can also compute the UV counterterms consistenly in $\overline{\mathrm{MS}}$, so that we can compute Feynman diagrams with local operators using only much simpler $L-1$ loop massless propagator intergrals. 
A first complication is that the $R^{*}$-operation cannot safely be applied after the Feynman rules are subsituted. An example of a problematic case is shown below:

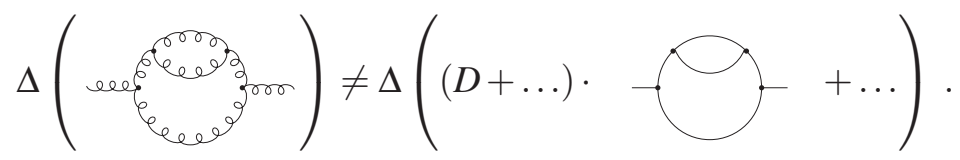

The $D$ that is created by the Feynman rules of the gluon does not commute with the (nested) $\Delta$ operation, since $\Delta$ truncates the Laurent series. In this example, the $D$ 'belongs' to the bubble subdiagram.

To get results in $\overline{\mathrm{MS}}$, we apply $R^{*}$ before the Feynman rules:

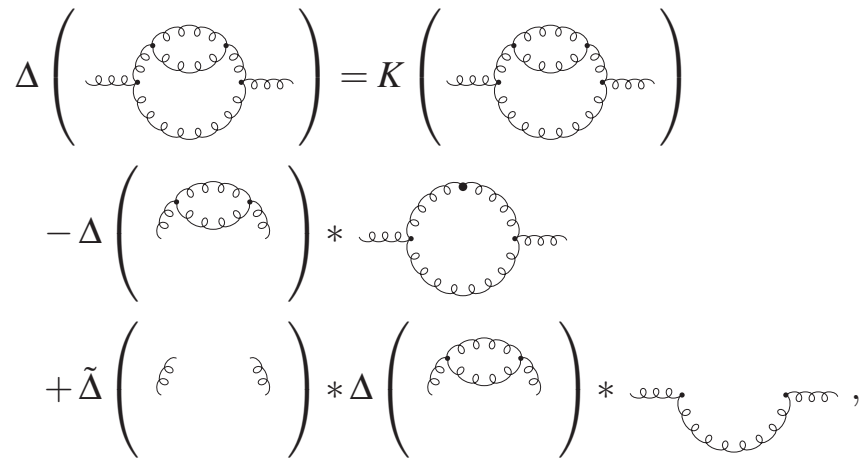

where $\tilde{\Delta}$ is the IR-counterterm operation [3]. Note that even though the subdiagrams have nontrivial vertex structures, the $R^{*}$-operation only requires the dimensionality of the vertices and propagators.

Now that the Feynman rules are only applied after the $R^{*}$ operation splits up the diagram into many subdiagrams, the bookkeeping becomes rather involved. The original connectivity needs to be remembered, and (dummy) indices arising from the Feynman rules need to be matched between components. An example is shown below:

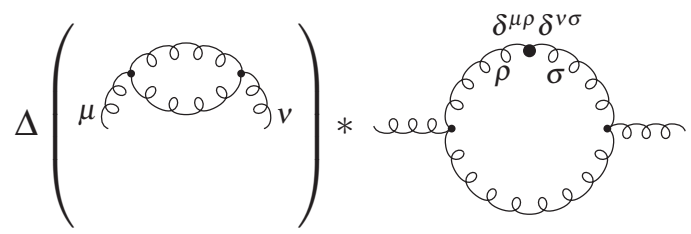

A second complication is that infrared rearrangement can only be applied on diagrams that are logarithmically divergent. A Taylor expansion can be used to make every diagram logarithmic. In the scalar case this is straightforward, but with non-trivial vertices and propagators we can essentially only apply the operator after the Feynman rules have been substituted. Thus, we have to bookkeep how the derivative should be taken. This leads to the introduction of new vertices, were some momenta are set to 0 , and to new propagators. 
Below we show a Taylor expansion in Q:

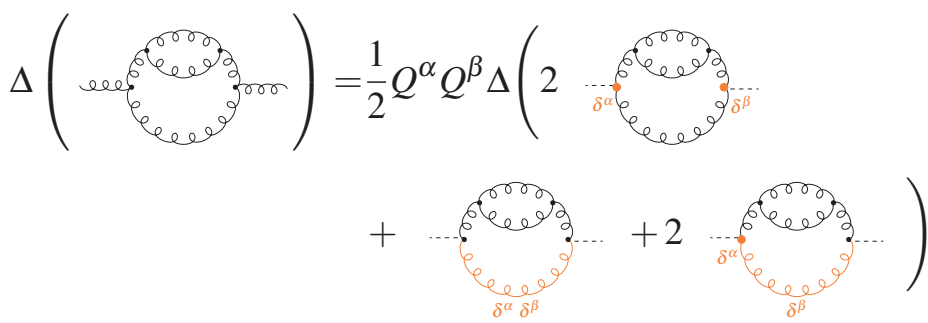

The $R^{*}$ can be applied on diagrams with derivatives acting on them without much change: the effect of a derivative is a decrease by one of the degree of the vertex/propagator.

Using the above setup, we can compute the UV counterterms safely in $\overline{\mathrm{MS}}$, using only massless $L-1$-loop propagator diagrams.

We have tested our new code by recomputing the five-loop beta function.

\section{Local operators}

As mentioned in the previous section, only the dimension of vertices and propagators is required to apply the $R^{*}$-operation. To compute the counterterms in $\overline{\mathrm{MS}}$, we have to use an abstract notation for the Feynman rules and potential derivatives. This means that it is straightforward to introduce local operators: they are simply special vertices.

We define a Qgraf-like notation for abstract propagators and vertices, where we code the momenta, the type of vertex or propagator, and the degree of UV divergence. For example, the following Higgs propagator graph

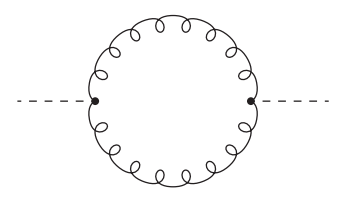

can be written as

$$
\begin{aligned}
& \operatorname{vx}(Q, p 1, p 2, \operatorname{vhg} g, 2) \star \\
& \operatorname{vx}(-p 1,-p 2,-Q, \operatorname{vhgg}, 2) \star \\
& \operatorname{prop}(p 1, g 1,-2) \star \operatorname{prop}(p 2, g 1,-2)
\end{aligned}
$$

To code derivatives in this convention, we add the path of the derivate through the vertex and generate dummy indices. For example, a derivative with respect to $Q$ through $p_{1}$ on the vertex

$\mathrm{vx}(\mathrm{Q}, \mathrm{p} 1, \mathrm{p} 2, \mathrm{vhg}, 2)$

becomes

$\mathrm{Q}(\mathrm{mu}) * \mathrm{vx}(\mathrm{Q} 0, \mathrm{p} 1, \mathrm{p} 2, \mathrm{vhgg}, 1, \mathrm{Q} 0, \mathrm{p} 1, \mathrm{mu})$

where $Q 0$ denotes that this momentum is set to 0 .

The workflow for computing UV counterterms can now be summarized in the following four steps: (1) the user defines Feynman rules and provides an abstract Feynman graph, (2) the $R^{*}$ program generates all abstract counterterms, (3) all the Feynman rules are evaluated and derivatives are taken, and (4) all resulting diagrams are computed with FORCER. 


\section{Summary and outlook}

We have sketched how the $R^{*}$-operation can be used to compute the poles of Feynman diagrams. Using these methods, we have computed the five-loop beta function for Yang-Mills theory with fermions [11] and the $R$-ratio and Higgs-boson decay widths $\Gamma_{H}$ to quarks and gluons [12]. We have briefly discussed the results for $\Gamma_{H \rightarrow g g}$ in the heavy-top limit.

Next, we have illustrated how to use the $R^{*}$-operation to compute UV counterterms in $\overline{\mathrm{MS}}$. Using an abstract notation for graphs and the fact that the $R^{*}$-operation only needs the dimensionality of propagators and vertices, we provide a framework to compute the UV counterterms of Feynman graphs with arbitrary local operators automatically. We have recomputed the five-loop QCD beta function to test the code.

Future work is to compute UV counterterms of theories with dimension six operators, and to apply the code to compute splitting functions. The latter is a computation of the complete pole part, but the use of the abstract notation can postpone a blow-up of the number of terms due to the application of the Feynman rules to a later stage in the program.

\section{Acknowledgements}

The research reported here has been supported by the European Research Council (ERC) Advanced Grant 320651, HEPGAME.

\section{References}

[1] K.G. Chetyrkin and F.V. Tkachov, Phys. Lett. B114 (1982) 340

[2] K.G. Chetyrkin and V.A. Smirnov, Phys. Lett. B144 (1984) 419

[3] F. Herzog and B. Ruijl, JHEP 05 (2017) 37, arXiv:1703.03776

[4] T. Ueda, B. Ruijl and J.A.M. Vermaseren, PoS LL2016 (2016) 070, arXiv:1607.07318

[5] B. Ruijl, T. Ueda and J.A.M. Vermaseren, https://doi.org/10.5281/zenodo.1043944

[6] B. Ruijl, T. Ueda and J.A.M. Vermaseren, arXiv:1704.06650

[7] J.A.M. Vermaseren, math-ph/0010025

[8] M. Tentyukov and J.A.M. Vermaseren, Comput. Phys. Commun. 181 (2010) 1419, hep-ph/0702279

[9] J. Kuipers, T. Ueda, J.A.M. Vermaseren and J. Vollinga, Comput. Phys. Commun. 184 (2013) 1453, arXiv: 1203.6543

[10] B. Ruijl, T. Ueda and J. Vermaseren, arXiv:1707.06453

[11] F. Herzog, B. Ruijl, T. Ueda, J.A.M. Vermaseren and A. Vogt, JHEP 02 (2017) 090, arXiv:1701.01404

[12] F. Herzog, B. Ruijl, T. Ueda, J.A.M. Vermaseren and A. Vogt, JHEP 08 (2017) 113, arXiv:1707.01044

[13] A.A. Vladimirov, Theor. Math. Phys. 43 (1980) 417

[14] P.A. Baikov, K.G. Chetyrkin and J.H. Kühn, Phys. Rev. Lett. 96 (2006) 012003, hep-ph/0511063

[15] P.A. Baikov, K.G. Chetyrkin, J.H. Kühn and J. Rittinger, Phys. Rev. Lett. 108 (2012) 222003, arXiv:1201.5804

[16] K. Chetyrkin, P. Baikov and J. Kühn, PoS LL2016 (2016) 010

[17] J. Davies, M. Steinhauser and D. Wellmann, Nucl. Phys. B920 (2017) 20, arXiv:1703.02988 